\title{
Hybrid Backscatter and Relaying Scheme for 6G Greencom IoT Networks with SWIPT
}

\author{
Sumit Gautam, Member IEEE, Shree Krishna Sharma, Senior Member IEEE, \\ Dinh-Hieu Tran, Student Member IEEE, Symeon Chatzinotas, \\ Senior Member IEEE, and Björn Ottersten, Fellow IEEE.
}

\begin{abstract}
In order to support the massive number of resource-constrained Internet of Things (IoT) devices and machine-type devices, it is crucial to design future beyond 5G/6G wireless networks in an energy-efficient manner. To this end, this letter proposes a novel two-hop hybrid backscatterand relaying scheme to facilitate simultaneous wireless information and power transfer (SWIPT) considering both the Time-Switching (TS) and Power-Splitting (PS) receiver architectures, while dynamically modelling the involved dual-hop time-period (TP) metric. An optimization problem is formulated for the joint optimization of throughput, harvested energy, and transmit power of a SWIPT-enabled system with the proposed hybrid scheme, and is solved using a nonlinear optimizer. Our numerical results in terms of weighted utility function show the superior performance of proposed hybrid scheme over backscatter-only and relay-only schemes, while also depicting their individual performance benefits over the corresponding benchmark SWIPT systems designed with the fixed-TP.
\end{abstract}

Index Terms

$5 \mathrm{G}$ and beyond/6G wireless networks, backscatter, greencom, IoT, relaying systems, SWIPT.

\section{INTRODUCTION}

The world witnesses the introduction of a novel generation of wireless communications in approximately every ten years. With the current speedy deployment of hardware for the fifth-

The authors are with the Interdisciplinary Centre for Security, Reliability and Trust (SnT), University of Luxembourg, L-1855 Luxembourg. (e-mail: \{sumit.gautam, shree.sharma, hieu.tran-dinh, symeon.chatzinotas, bjorn.ottersten\}@uni.lu).

Corresponding author: Sumit Gautam (e-mail: sumit.gautam@uni.lu). 
generation $(5 \mathrm{G})$ of mobile wireless systems in 2020 , we are now at a juncture to anticipate what lies ahead for the constitution of the sixth generation (6G) mobile cellular systems [1]. The incoming crucial updates to the hardware equipment for 5G may require dense infrastructure deployment [2]. In future, it will become important to leverage the $5 \mathrm{G}$ architecture for internetof-things (IoT) systems, along with the introduction of some methods to increase the coverage area as well as a consideration of energy harvesting (EH) frameworks comprising the concept of Green Communications (Greencom)-based IoT systems.

To enhance the network coverage of wireless communications systems, certain well-known methods such as backscattering and/or relaying have shown immense potential [3]. A backscatter is a battery-less device used to modify and reflect the transmit signal (with encoded data) towards the desired user, where it may be utilized to energize the IoT devices as well as to enable the deployment of battery-free devices in future networks. In case of relaying, the transmitted signal from the source is received at the relay device and re-transmitted to the end-user after either a direct amplification according to the amplify-and-forward (AF) protocol [4], or a decoding or quantization process before its re-transmission as a re-encoded signal with message suppression, according to the decode-and-forward (DF) protocol [5]. Regarding the facilitation of $\mathrm{EH}$ at the end-user, simultaneous wireless information and power transfer (SWIPT) technique seems promising [6]. In this regard, the power-splitting (PS) and time-switching (TS) schemes have been widely considered to deploy SWIPT receivers in practice.

Recent developments in the area of backscatter-and-relaying scheme have focused on important aspects such as cooperation enhancement, however, with the consideration of a linear EH model [7]. In [8], the authors presented an interesting framework for wireless energy beamforming in the backscatter-and-relay based communications system, wherein a non-linear EH mechanism is considered at the intermediary device but not at the end-user. Additionally, most related works focus on the maximization of either the signal-to-noise ratio (SNR) [9], or the throughput [10]. However, it is important to optimize the joint metrics of data throughput, harvested energy and transmit power of the SWIPT-enabled 6G systems. On one hand, it is noteworthy that the backscatter-only mechanism may not be suitable for dense deployment scenarios [11]. Whereas on the other hand, the power-dependence of relays is critical in the relaying-only scenarios, wherein a power-failure may lead to the collapse of whole wireless system without any backup. In this regard, hybrid backscatter-and-relaying may ensure a sustainable wireless ecosystem 
where both devices may supplement or complement each other accordingly. Moreover, in the context of the hybrid framework, a comparative study between the PS and TS SWIPT schemes with non-linear EH model along with the design of dynamic dual-hop time-period (TP) has not been considered for investigation in the literature so far.

In this letter, we present a dual-hop system wherein a transmit source transfers both information and energy to a SWIPT-enabled end-user employing either PS or TS architecture. We investigate three distinct Greencom systems incorporating a backscatter node, a relaying node, and a novel backscatter-and-relaying node, respectively; which either reflects and/or re-transmits the information-and-energy signal to the end-user, in addition to the weak direct link. The novelty and main contributions of this work are listed as follows.

(i) In contrast to the existing backscatter-only and relaying-only techniques, we propose a novel hybrid backscatter-and-relaying scheme to facilitate SWIPT to a PS- or TS-enabled end-user, along with the dynamic designing of dual-hop TP.

(ii) We formulate a novel problem (incorporating the three systems) to maximize the weighted utility function comprising data throughput, harvested energy and transmit power, subjected to some quality-of-service (QoS) constraints. Unlike the other works with assumption of equal timeintervals in the two hops (also considered herein as the benchmarks), we present a framework to dynamically design the TP for the dual-hop link, along with the computation of PS or TS factors.

(iii) The effectiveness of the proposed hybrid backscatter-and-relaying scheme is shown over the backscatter-only and relay-only schemes for both PS and TS SWIPT schemes via numerical analysis, with individual benefits shown over their respective benchmark designs having a fixed dual-hop TP.

The remainder of this paper is organized as follows. Section II presents a description of the system model and Section III provides an analysis of the considered Greencom networks. Section IV focuses on the joint optimization of data throughput, harvested energy and transmit power. Section V presents the simulation results. Finally, Section VI concludes the paper.

\section{System Model}

We consider three wireless Greencom network scenarios for investigation, with a single transmit source, a single reflecting and/or re-transmission device (i.e., either backscatter or relay 


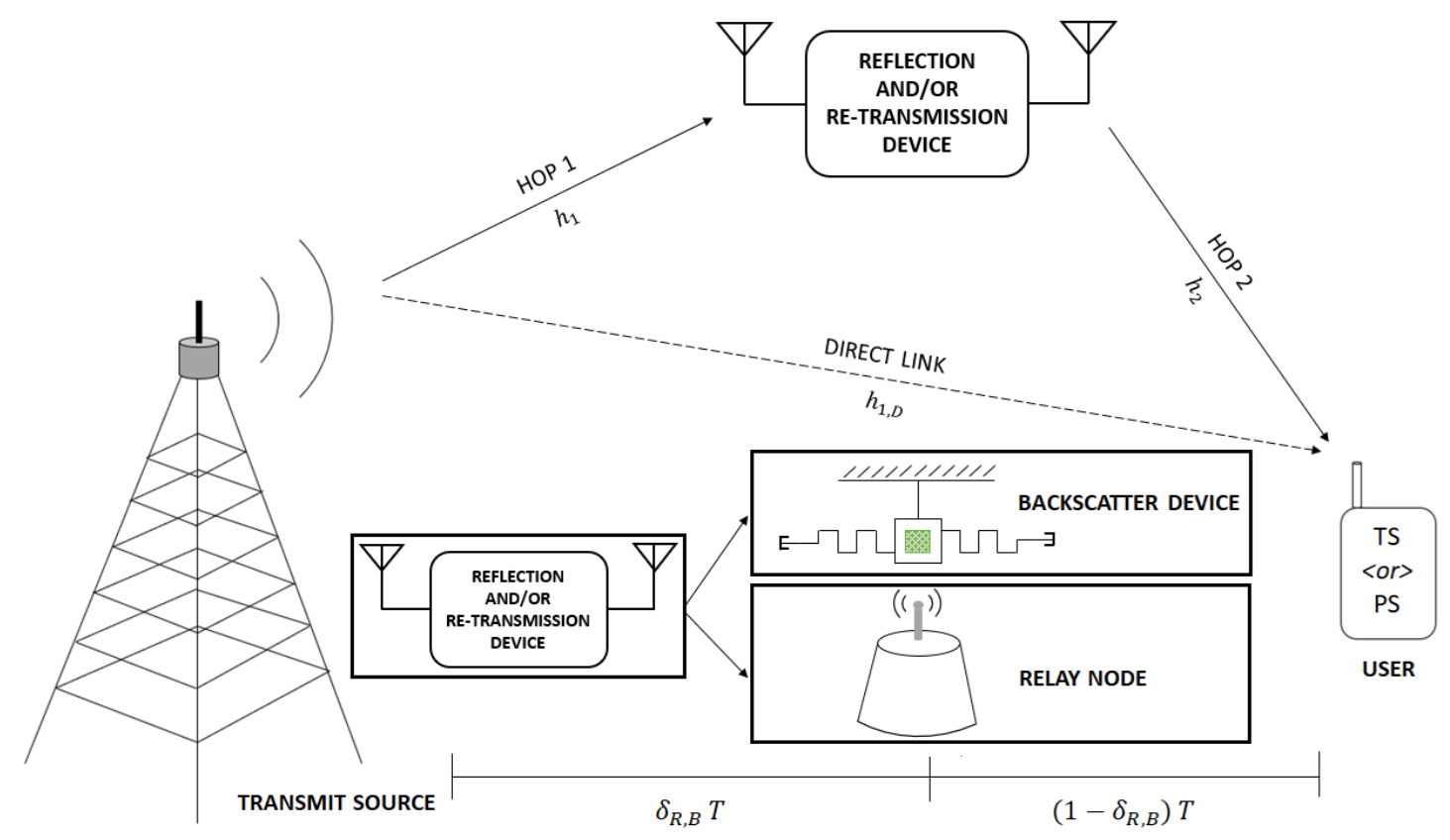

Fig. 1: Dual hop system with a backscatter and/or relaying device.

or both, respectively), and a single user at the receiving end, as represented in Fig. 1. The transmit source communicates with the end-user via two communication links within a single time-slot. In particular, besides the conventional direct link, we assume the availability of a couple of intermediary links to assist in the delivery of the desired transmit signal. The end-user is assumed to be capable of performing both information decoding (ID) and energy harvesting (EH) simultaneously.

Let us define $P_{\mathcal{T}}$ as the transmit power at the source, $\mathcal{B}$ as the overall bandwidth, and $\mathrm{T}$ as the TP for further analysis. In each system, the direct link is assumed to be active throughout the TP, with a pre-defined channel coefficient of $h_{1, D} \cdot{ }^{1}$ The transmit source emits the signal intended for the corresponding SWIPT-enabled end-user. The process is carried out using a direct link through the whole TP, with additional dual-hop assistance via a reflecting and/or re-transmitting device over the same TP. More specifically, the source transmits a symbol $s \in \mathbb{C}$, which is received by the destination and the reflecting and/or re-transmitting device. Without loss of generality, we assume $\mathbb{E}\left[|s|^{2}\right]=1$, with operation $\mathbb{E}[\cdot]$ denoting the expectation value. The signal received

\footnotetext{
${ }^{1}$ For analytical convenience, we assume that the channel state information (CSI) is known to the intended devices.
} 
by the end-user via direct link is defined as: $y_{1, D}=\sqrt{P_{\mathcal{T}}} h_{1, D} s+n_{u_{a}}$, where $n_{u_{a}}$ is the additive white Gaussian noise (AWGN) at the end-user which is an independent and identically distributed (i.i.d.) complex Gaussian random variable with zero mean and variance $\sigma_{n_{u_{a}}}^{2}$.

Concerning the SWIPT-enabled receiver device, two different SWIPT schemes namely PS or TS, respectively, may be adopted. For the PS scheme, an optimal fraction of the received signal is provided to the information decoder and the remaining part is provided to the energy harvester. In this case, the PS ratio is denoted by $\rho$, where $0 \leq \rho \leq 1$. Alternatively, when the receiver incorporates the TS scheme, we define a time switching ratio, $\tau$, where $0 \leq \tau \leq 1$. In particular, for the first fraction of time period, all the received signal power is used for harvesting energy, whereas during the remaining time, information decoding from the received signal takes place.

In order to estimate the amount of harvested energy at the end-user, we adopt the sigmoidal function based non-linear EH model [12], [13], defined as

$$
\mathcal{E}(x)=\frac{\mathcal{E}^{\prime}}{1-\phi} \cdot\left(\frac{1}{1+e^{(-\alpha x+\alpha \beta)}}-\phi\right),
$$

where $x$ is the input power at the energy harvesting module, $\phi \triangleq \frac{1}{1+\exp (\alpha \beta)}$, the constant $\mathcal{E}^{\prime}$ is obtained by determining the maximum harvested energy on the saturation of the energy harvesting circuit, and $\alpha$ and $\beta$ are specific to the capacitor and diode turn-on voltage metrics at the $\mathrm{EH}$ circuit. The harvested energy using the PS and TS schemes are respectively given by

$$
\begin{gathered}
\mathcal{E}^{\mathrm{PS}}(x, \rho)=\frac{\mathcal{E}^{\prime}}{1-\phi} \cdot\left(\frac{1}{1+e^{(-\alpha \rho x+\alpha \beta)}}-\phi\right), \\
\mathcal{E}^{\mathrm{TS}}(x, \tau)=\tau \cdot \frac{\mathcal{E}^{\prime}}{1-\phi} \cdot\left(\frac{1}{1+e^{(-\alpha x+\alpha \beta)}}-\phi\right) .
\end{gathered}
$$

The energy harvested at the end-user using the PS and TS schemes via direct link signal is given by $\mathcal{E}_{1, D}^{\mathrm{PS}}\left(\mathbb{E}\left[\left|y_{1, D}\right|^{2}\right], \rho\right)$ and $\mathcal{E}_{1, D}^{\mathrm{TS}}\left(\mathbb{E}\left[\left|y_{1, D}\right|^{2}\right], \tau\right)$, computed according to (2) and (3), respectively. Further, we assume that the normalized time slots use the terms power and energy interchangeably.

The effective signal-to-noise ratio (SNR) via direct link as seen at the ID branch of the enduser, respectively for PS and TS schemes, is given by

$$
\Upsilon_{1, D}^{\mathrm{PS}}=\frac{(1-\rho) P_{\mathcal{T}}\left|h_{1, D}\right|^{2}}{(1-\rho) \sigma_{n_{u_{a}}}^{2}+\sigma_{n_{u_{I}}}^{2}} \text {, and } \Upsilon_{1, D}^{\mathrm{TS}}=\frac{P_{\mathcal{T}}\left|h_{1, D}\right|^{2}}{\sigma_{n_{u_{a}}}^{2}+\sigma_{n_{u_{I D}}}^{2}}
$$

where $n_{u_{I D}} \in \mathbb{C} \mathbb{N}\left(0, \sigma_{n_{u_{I D}}}^{2}\right)$ is the the noise introduced by the baseband processing circuit. The 
effective throughput achieved at the end-user, combining the PS and TS schemes is given by

$$
R_{1, D}=\left\{\begin{array}{l}
R_{1, D}^{\mathrm{PS}}=\mathcal{B} \log _{2}\left(1+\Upsilon_{1, D}^{\mathrm{PS}}\right) \\
R_{1, D}^{\mathrm{TS}}=(1-\tau) \mathcal{B} \log _{2}\left(1+\Upsilon_{1, D}^{\mathrm{TS}}\right)
\end{array} .\right.
$$

\section{ANALYSIS OF GREENCOM NETWORK SCENARIOS}

In this section, we present the discussion on the considered Greencom network scenarios in detail. To proceed, we define the dynamic time-splitting (DTS) ratio as $\delta_{B R}$, where $0<\delta_{B R} \leq 1$, to assist the dual-hop mechanism. Specifically, we assume that the first-hop spans over $\delta_{B R} T$ of the TP while in the second hop, the remainder of the TP, i.e., $\left(1-\delta_{B R}\right) \mathrm{T}$, is allocated for successful reflection and/or re-transmission of the intended signal from the backscatter and/or relay device(s) to the end-user.

\section{A. Traditional backscatter device-based or relay-based system}

In this case, we assume the availability of either a single antenna backscatter device as the reflecting node or a single antenna relaying node as a re-transmission device on the second hop. Further, we refer the two above-mentioned systems as backscatter device-based Greencom network (BGN) and relaying device-based Greencom network (RGN), respectively. The channel coefficient in the first phase is defined as $h_{1}$.We represent the signal seen at the reflecting/retransmitting device as: $r_{1}=\sqrt{P_{\mathcal{T}}} h_{1} s+n_{1}$, where $n_{1}$ is the AWGN introduced due to the backscatter/relay, which is an i.i.d. complex Gaussian random variable with zero mean and variance $\sigma_{n_{1}}^{2}$. In this context, we express $\left(r_{1}, h_{1}, n_{1}\right):=\left\{\left(r_{1, B}, h_{1, B}, n_{B}\right):\right.$ for BGN; $\left(r_{1, R}, h_{1, R}, n_{R}\right)$ : for $\mathrm{RGN}$.

The SNR and the effective data throughput expressions are respectively defined as

$$
\Upsilon_{1}=\frac{P_{\mathcal{T}}\left|h_{1}\right|^{2}}{\sigma_{n_{1}}^{2}} \text {, and } R_{1}=\delta_{B R} \mathcal{B} \log _{2}\left(1+\Upsilon_{1}\right),
$$

where $\left(\Upsilon_{1}, R_{1}, \sigma_{n_{1}}^{2}\right):=\left\{\left(\Upsilon_{1, B}, R_{1, B}, \sigma_{n_{B}}^{2}\right)\right.$ : for BGN; $\left(\Upsilon_{1, R}, R_{1, R}, \sigma_{n_{R}}^{2}\right):$ for RGN $\}$.

In the second hop, the channel coefficient is defined as $h_{2}$. In case of BGN, the intended signal from the backscatter is reflected to the end-user with the corresponding efficiency coefficient of $\sqrt{\eta}$, where $0<\sqrt{\eta} \leq 1$. Whereas in the case of RGN, the relay re-transmits the signal after scaling it by a complex amplification coefficient $w$. In order to ensure feasibility of the RGB system, we impose an upper bound on the total relay power defined by: $0<|w|^{2} \leq P_{R}$, where 
$P_{R}=\frac{P^{\star}-P_{\mathcal{T}}}{P_{\mathcal{T}}\left|h_{1, R}\right|^{2}+\sigma_{n_{R}}^{2}}$ is the maximum overall available power at the relay, and the transmitter-relay system is bounded by an overall power of $P^{\star}$, such that $P^{\star}>\max \left(P_{\mathcal{T}}, P_{R}\right)$. To proceed, we define the following metric:

$$
\Xi=\left\{\begin{array}{l}
\sqrt{\eta}: \text { Backscatter Coefficient in BGN } \\
w: \text { Relay Amplification Coefficient in RGN }
\end{array} .\right.
$$

The received signal as seen at the end-user via reflection or re-transmission from the backscatter or relay device, respectively, is represented as $y_{2}=\Xi h_{2} r_{1}+n_{u_{a}}$, where $\left(y_{2}, h_{2}, r_{1}\right):=$ $\left\{\left(y_{2, B}, h_{2, B}, r_{1, B}\right)\right.$ : for BGN; $\left(y_{2, R}, h_{2, R}, r_{1, R}\right)$ : for $\left.\mathrm{RGN}\right\}$.

We define the SNR obtained at the ID block of the end-user via second hop link, according to the PS and TS schemes, as

$$
\begin{gathered}
\Upsilon_{2}^{\mathrm{PS}}=\frac{|\Xi|^{2}(1-\rho) P_{\mathcal{T}}\left|h_{1}\right|^{2}\left|h_{2}\right|^{2}}{(1-\rho)\left(|\Xi|^{2} \sigma_{n_{1}}^{2}+\sigma_{n_{u_{a}}}^{2}\right)+\sigma_{n_{u_{I D}}}^{2}}, \\
\Upsilon_{2}^{\mathrm{TS}}=\frac{|\Xi|^{2} P_{\mathcal{T}}\left|h_{1}\right|^{2}\left|h_{2}\right|^{2}}{|\Xi|^{2} \sigma_{n_{1}}^{2}+\sigma_{n_{u_{a}}}^{2}+\sigma_{n_{u_{I}}}^{2}},
\end{gathered}
$$

where $\left(\Upsilon_{2}^{\mathrm{PS}}, \Upsilon_{2}^{\mathrm{TS}}, h_{1}, h_{2}, \sigma_{n_{1}}^{2}\right):=\left\{\left(\Upsilon_{2, B}^{\mathrm{PS}}, \Upsilon_{2, B}^{\mathrm{TS}}, h_{1, B}, h_{2, B}, \sigma_{n_{B}}^{2}\right):\right.$ for BGN; $\left(\Upsilon_{2, R}^{\mathrm{PS}}, \Upsilon_{2, R}^{\mathrm{TS}}, h_{1, R}, h_{2, R}\right.$, $\left.\sigma_{n_{R}}^{2}\right)$ : for RGN\}. The effective throughput achieved at the end-user, incorporating the respective PS and TS schemes is defined as

$$
R_{2}=\left\{\begin{array}{l}
R_{2}^{\mathrm{PS}}=\left(1-\delta_{B R}\right) \mathcal{B} \log _{2}\left(1+\Upsilon_{2}^{\mathrm{PS}}\right) \\
R_{2}^{\mathrm{TS}}=(1-\tau)\left(1-\delta_{B R}\right) \mathcal{B} \log _{2}\left(1+\Upsilon_{2}^{\mathrm{TS}}\right)
\end{array},\right.
$$

where $\left(R_{2}, R_{2}^{\mathrm{PS}}, \Upsilon_{2}^{\mathrm{PS}}, R_{2}^{\mathrm{TS}}, \Upsilon_{2}^{\mathrm{TS}}\right):=\left\{\left(R_{2, B}, R_{2, B}^{\mathrm{PS}}, \Upsilon_{2, B}^{\mathrm{PS}}, R_{2, B}^{\mathrm{TS}}, \Upsilon_{2, B}^{\mathrm{TS}}\right)\right.$ : for $\mathrm{BGN} ;\left(R_{2, R}, R_{2, R}^{\mathrm{PS}}, \Upsilon_{2, R}^{\mathrm{PS}}\right.$, $\left.R_{2, R}^{\mathrm{TS}}, \Upsilon_{2, R}^{\mathrm{TS}}\right):$ for $\left.\mathrm{RGN}\right\}$.

The PS and TS expressions for harvested energy are denoted as $\mathcal{E}_{2}^{\mathrm{PS}}\left(\mathbb{E}\left[\left|y_{2}\right|^{2}\right], \rho\right)$ and $\mathcal{E}_{2}^{\mathrm{TS}}\left(\mathbb{E}\left[\left|y_{2}\right|^{2}\right], \tau\right)$, computed according to (2) and (3), respectively, where $\left(\mathcal{E}_{2}^{\mathrm{PS}}, \mathcal{E}_{2}^{\mathrm{TS}}, y_{2}\right):=\left\{\left(\mathcal{E}_{2, B}^{\mathrm{PS}}\right.\right.$, $\left.\mathcal{E}_{2, B}^{\mathrm{TS}}, y_{2, B}\right)$ : for $\mathrm{BGN} ;\left(\mathcal{E}_{2, R}^{\mathrm{PS}}, \mathcal{E}_{2, R}^{\mathrm{TS}}, y_{2, R}\right)$ : for $\left.\mathrm{RGN}\right\}$.

At the end-user, the overall data throughput achieved incorporating the yield from the second hop and direct link, for respective PS and TS schemes, is defined as

$$
R_{U}=\left\{\begin{array}{l}
R_{U}^{\mathrm{PS}}=R_{1, D}^{\mathrm{PS}}+R_{2}^{\mathrm{PS}} \\
R_{U}^{\mathrm{TS}}=R_{1, D}^{\mathrm{TS}}+R_{2}^{\mathrm{TS}}
\end{array},\right.
$$

where $\left(R_{U}, R_{U}^{\mathrm{PS}}, R_{U}^{\mathrm{TS}}\right):=\left\{\left(R_{U, B}, R_{U, B}^{\mathrm{PS}}, R_{U, B}^{\mathrm{TS}}\right):\right.$ for BGN; $\left(R_{U, R}, R_{U, R}^{\mathrm{PS}}, R_{U, R}^{\mathrm{TS}}\right)$ : for RGN $\}$. The 
effective harvested energy expression incorporating both PS and TS schemes is:

$$
E_{U}=\left\{\begin{array}{l}
E_{U}^{\mathrm{PS}}=\mathcal{E}_{1, D}^{\mathrm{PS}}\left(\mathbb{E}\left[\left|y_{1, D}\right|^{2}\right], \rho\right)+\left(1-\delta_{B R}\right) \mathcal{E}_{2}^{\mathrm{PS}}\left(\mathbb{E}\left[\left|y_{2}\right|^{2}\right], \rho\right) \\
E_{U}^{\mathrm{TS}}=\mathcal{E}_{1, D}^{\mathrm{TS}}\left(\mathbb{E}\left[\left|y_{1, D}\right|^{2}\right], \tau\right)+\left(1-\delta_{B R}\right) \mathcal{E}_{2}^{\mathrm{TS}}\left(\mathbb{E}\left[\left|y_{2}\right|^{2}\right], \tau\right)
\end{array}\right.
$$

where $\left(E_{U}, E_{U}^{\mathrm{PS}}, E_{U}^{\mathrm{TS}}\right):=\left\{\left(E_{U, B}, E_{U, B}^{\mathrm{PS}}, E_{U, B}^{\mathrm{TS}}\right)\right.$ : for BGN; $\left(E_{U, R}, E_{U, R}^{\mathrm{PS}}, E_{U, R}^{\mathrm{TS}}\right)$ : for RGN $\}$.

\section{B. Proposed Hybrid Backscatter and Relaying Scheme}

We present herein the proposed relaying in Greencom networks with backscatter (RGB) scheme to assist in facilitating SWIPT to the end-user over the second hop. In this case, the hybrid backscatter-and-relaying node receives the transmit signal on both the backscatter and relay systems equipped with single antennas each, over the above-defined channel coefficients of $h_{1, B}$ and $h_{1, R}$, respectively. We define the DTS ratio for the first hop as $\delta_{B R}$, where $0<\delta_{B R} \leq 1$. The signals, SNR and throughput expressions seen at the respective backscatter and relay nodes are similar to the ones defined in the previous sections. ${ }^{2}$

Over the second hop, the backscatter device uses the coefficient $\sqrt{\eta}$ to reflect the transmit signal while the relay employs AF-protocol to boost the transmit signal with the help of $w$ and retransmit the same to the end-user over the second hop. For the remainder of TP, i.e., $\left(1-\delta_{B R}\right) \mathrm{T}$, the re-directed signals from the backscatter and relay experiences channel coefficients of $h_{2, B}$ and $h_{2, R}$, respectively. The corresponding signals, SNR and throughput expressions are similar as defined before. The overall effective throughput and the harvested energy expressions achieved at the end-user, incorporating the PS and TS schemes are respectively expressed as

$$
\begin{gathered}
R_{U, B R}=\left\{\begin{array}{l}
R_{U, B R}^{\mathrm{PS}}=R_{1, D}^{\mathrm{PS}}+R_{2, B}^{\mathrm{PS}}+R_{2, R}^{\mathrm{PS}} \\
R_{U, B R}^{\mathrm{TS}}=R_{1, D}^{\mathrm{TS}}+R_{2, B}^{\mathrm{TS}}+R_{2, R}^{\mathrm{TS}}
\end{array}\right. \\
E_{U, B R}=\left\{\begin{aligned}
E_{U, B R}^{\mathrm{PS}}= & \mathcal{E}_{1, D}^{\mathrm{PS}}\left(\mathbb{E}\left[\left|y_{1, D}\right|^{2}\right], \rho\right)+\left(1-\delta_{B R}\right) \mathcal{E}_{2, B}^{\mathrm{PS}}\left(\mathbb{E}\left[\left|y_{2, B}\right|^{2}\right], \rho\right) \\
+ & \left(1-\delta_{B R}\right) \mathcal{E}_{2, R}^{\mathrm{PS}}\left(\mathbb{E}\left[\left|y_{2, R}\right|^{2}\right], \rho\right) \\
E_{U, B R}^{\mathrm{TS}}= & \mathcal{E}_{1, D}^{\mathrm{TS}}\left(\mathbb{E}\left[\left|y_{1, D}\right|^{2}\right], \tau\right)+\left(1-\delta_{B R}\right) \mathcal{E}_{2, B}^{\mathrm{TS}}\left(\mathbb{E}\left[\left|y_{2, B}\right|^{2}\right], \tau\right) \\
& +\left(1-\delta_{B R}\right) \mathcal{E}_{2, R}^{\mathrm{TS}}\left(\mathbb{E}\left[\left|y_{2, R}\right|^{2}\right], \tau\right)
\end{aligned}\right.
\end{gathered}
$$

\footnotetext{
${ }^{2}$ Note that the backscatter and relay devices in the proposed RGB scheme may be co-located or not. However, for analytic convenience related to the DTS design, we assume a co-located hybrid backscatter-and-relaying set-up.
} 


\begin{tabular}{|c||c|c|c|c|c|c|c|}
\hline System Parameter & $P$ & $R_{\mathrm{I}}$ & $R_{\mathrm{II}}$ & $R_{U}$ & $E_{U}$ & $\delta$ & $\widetilde{w}$ \\
\hline \hline $\mathrm{BGN}$ & $P_{\mathcal{T}}$ & $R_{1, B}$ & $R_{2, B}$ & $R_{U, B}$ & $E_{U, B}$ & $\delta_{B R}$ & - \\
\hline $\mathrm{RGN}$ & $P_{\mathcal{T}}+P_{R}$ & $R_{1, R}$ & $R_{2, R}$ & $R_{U, R}$ & $E_{U, R}$ & $\delta_{B R}$ & $w$ \\
\hline $\mathrm{RGB}$ & $P_{\mathcal{T}}+P_{R}$ & {$\left[\begin{array}{l}R_{1, B} \\
R_{1, R}\end{array}\right]$} & {$\left[\begin{array}{l}R_{2, B} \\
R_{2, R}\end{array}\right]$} & $R_{U, B R}$ & $E_{U, B R}$ & $\delta_{B R}$ & $w$ \\
\hline
\end{tabular}

TABLE I: Parameter definitions to assist the problem formulation.

\section{Problem Formulation And Solution}

Herein, we present the problem to jointly optimize the data throughput, harvested energy and transmit power, subjected to QoS constraints for each considered schemes (viz., BGN, RGN, and RGB). Before proceeding to the main hypothesis, we introduce some variables to simplify the problem representation.

\section{A. Variable definitions to assist the problem formulation}

In this section, we define some additional parameters corresponding to the overall transmit power, data throughput and harvested energy expressions obtained at the end-user, the DTS ratios, the relay amplification factor and the TS and PS ratios; to refer to the aforementioned frameworks of BGN, RGN, and RGB altogether. In this context, the variables are sequentially defined in Table I.

We additionally consider another parameter to assist the PS and TS ratio metrics, defined as follows.

$$
\theta:=\{\rho: \text { for PS Scheme; } \tau: \text { for TS Scheme }\}
$$

It is clear that the newly defined parameters will adopt values according to the type of scenario chosen. Thus, we mathematically formulate the optimization problem in the following while incorporating the parameters defined in Table I and (13).

\section{B. Optimization problem with weighted utility function}

We formulate the problem with weighted utility function to jointly optimize the data throughput, harvested energy and total transmit power of the source, while ensuring that the demanded throughput and the harvested power at the destination node are both individually above the given 


\begin{tabular}{|c|c|c|c|}
\hline$F\left(P_{\mathcal{T}}, \delta, \theta, \widetilde{w}\right)$ & $G\left(P_{\mathcal{T}}, \delta, \theta, \widetilde{w}\right)$ & $H\left(P_{\mathcal{T}}, \delta, \theta, \widetilde{w}\right)$ & $I\left(P_{\mathcal{T}}, \delta, \theta, \widetilde{w}\right)$ \\
\hline$\frac{\omega_{R} R_{U}+\omega_{E} E_{U}}{\omega_{P} P_{\mathcal{T}}}$ & $R_{\mathrm{II}}-R_{\mathrm{I}} \leq 0$ & $\varsigma-R_{U} \leq 0$ & $\xi-E_{U} \leq 0$ \\
\hline
\end{tabular}

TABLE II: Definitions to comprise the Lagrangian expression.

thresholds. The overall optimization problem incorporating the BGN, RGN and RGB systems is analytically represented as

$$
\begin{aligned}
&(P 1): \max _{P_{\mathcal{T}, \delta, \theta, \widetilde{w}}} \frac{\omega_{R} R_{U}+\omega_{E} E_{U}}{\omega_{P} P_{\mathcal{T}}} \\
& \text { subject to : } \quad(C 1): R_{\mathrm{I}} \geq R_{\mathrm{II}}, \quad(C 2): R_{U} \geq \varsigma, \\
& \\
&(C 3): E_{U} \geq \xi, \quad(C 4): 0 \leq \delta \leq 1, \\
& \\
&(C 5): 0 \leq \theta \leq 1,(C 6): 0<P \leq P^{\star}, \\
&(C 7)^{*}: 0 \leq|\widetilde{w}|^{2} \leq P_{R},
\end{aligned}
$$

where $\varsigma$ and $\xi$ are respectively the minimum demanded throughput and harvested energy by the destination SWIPT-capable user, $\omega_{R}, \omega_{E}$ and $\omega_{P}$ are the constant weighing factors corresponding to the data throughput, harvested energy and the transmit power, respectively. We use $\theta$ to interchangeably refer to the PS or TS splitting factor $\rho$ or $\tau$, respectively. In order to ensure feasibility during the second hop transmission, we make use of $(C 1)$. The constraints $(C 2)$ and $(C 3)$ are the minimum thresholds on the overall data rate and overall harvested energy, respectively. The upper and lower bounds of the DTS fraction and the SWIPT splitting factors are represented in $(C 4)$ and $(C 5)$ respectively. The constraint with maximum transmit power budget of the system is incorporated using $(C 6)$. Note that the variable $\widetilde{w}$ and $(C 7)^{*}$ are considered only during the analysis of RGN and RGB systems. In the following, we analyse $(P 1)$ and seek a suitable solution.

\section{Solution to the above-mentioned problem}

The problem $(P 1)$ is difficult to solve, since it has a non-convex form for both TS and PS schemes. In order to obtain an analytical solution for the joint objective optimization problem in $(P 1)$, we assume $\mathcal{D}$ as the set of all possibilities of $P_{\mathcal{T}}, \delta, \theta$ and $\widetilde{w}$ which satisfy the constraints 
$(C 4)-(C 7)$. Then the corresponding Lagrange dual function is written as

$$
J(\boldsymbol{\Lambda}) \triangleq \max _{\left\{P_{\mathcal{T}}, \delta, \theta, \widetilde{w}\right\} \in \mathcal{D}} \mathcal{L}\left(P_{\mathcal{T}}, \delta, \theta, \widetilde{w} ; \boldsymbol{\Lambda}\right),
$$

where the Lagrangian is expressed as

$$
\begin{gathered}
\mathcal{L}\left(P_{\mathcal{T}}, \delta, \theta, \widetilde{w} ; \boldsymbol{\Lambda}\right)=F\left(P_{\mathcal{T}}, \delta, \theta, \widetilde{w}\right)-\lambda_{1} G\left(P_{\mathcal{T}}, \delta, \theta, \widetilde{w}\right) \\
-\lambda_{2} H\left(P_{\mathcal{T}}, \delta, \theta, \widetilde{w}\right)-\lambda_{3} I\left(P_{\mathcal{T}}, \delta, \theta, \widetilde{w}\right),
\end{gathered}
$$

with $\boldsymbol{\Lambda}=\left(\lambda_{1}, \lambda_{2}, \lambda_{3}\right) \succeq 0$ denoting the vector of the dual variables associated with the constraints $(C 1)-(C 3)$, respectively, while the related functions are as defined in Table II. The dual optimization problem is hence represented as

$$
\begin{array}{cl}
(P 2): \min _{\boldsymbol{\Lambda}} J(\boldsymbol{\Lambda}) \\
\text { subject to : } \boldsymbol{\Lambda} \succeq 0 .
\end{array}
$$

Since it is explicit that a dual problem is always convex by definition [14], therefore the gradient or subgradient-based methods can be used to minimize $J(\boldsymbol{\Lambda})$ with guaranteed convergence. In this regard, an alternating optimization of the involved parameters is performed in an iterative manner, until the convergence of the weighted utility function value. The intermediary closed-form expressions for the involved parameters may be obtained using the Karush-Kuhn-Tucker (KKT) conditions [14]. Discussion on the obtained numerical solutions is provided in the succeeding section.

\section{Simulation Results}

In this section, we present a comparative study among the RGB, RGN and BGN systems. The three cases are analyzed using the MATLAB R2019b, with optimization performed via fmincon $(\cdot)$ solver present in the optimization toolbox [15], where the solutions are obtained with the help of interior point algorithm [14]. We assume an ITU-R outdoor framework (site-general model for propagation within street canyons) [16] to generate channel realizations with the path-loss exponent:

$$
P L(d, f)=10 a \log _{10}(d)+b+10 c \log _{10}(f)+N(0, \sigma) \mathrm{dB},
$$

where $d$ is 3D direct distance between the transmitting and receiving stations (m), $f$ is the operating frequency $(\mathrm{GHz})$, the coefficients $a, b$, and $c$ are associated with the increase of the 


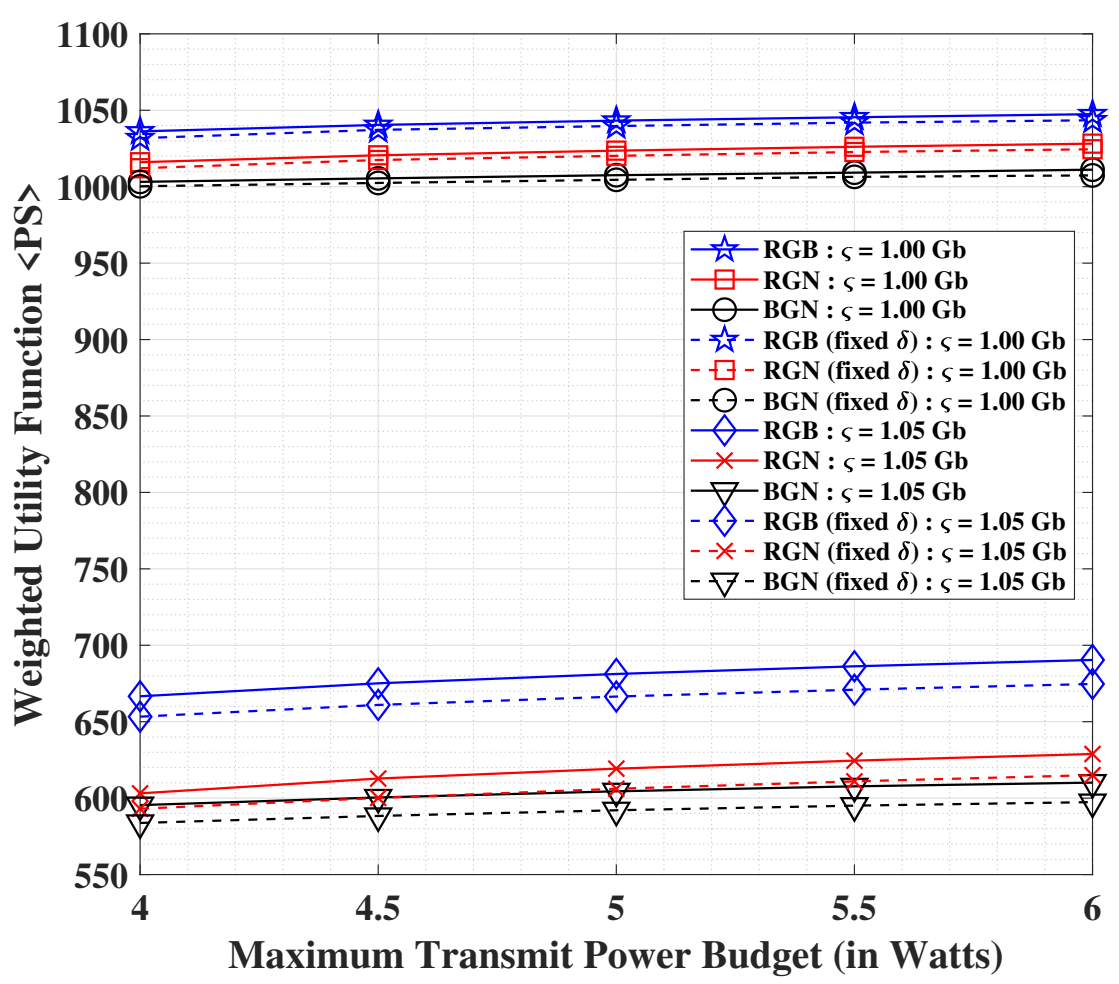

Fig. 2: Performance analysis of weighted utility function for PS scheme versus the maximum limitation on the total transmit power.

path loss with distance, the offset value of the path loss, and the increase of the path loss with frequency, respectively, and $N(0, \sigma)$ is a zero mean Gaussian random variable with a standard deviation $\sigma(\mathrm{dB})$. The channels coefficients for the direct link and the dual-hop links are generated accordingly. Specifically, we choose: $f=24 \mathrm{GHz}, d$ is randomly chosen between $8 \mathrm{~m}$ and 10 $\mathrm{m}$ in case of backscatter/relaying device and randomly between $12 \mathrm{~m}$ and $15 \mathrm{~m}$ for the end-user with respect to the transmitter, $a=2.12, b=29.2, c=2.11$ and $\sigma=5.06 \mathrm{~dB}$. The simulation results presented in this section assume an overall bandwidth of $B=50 \mathrm{MHz}$. We set $\sigma_{n_{B}}^{2}=\sigma_{n_{R}}^{2}$ $=\sigma_{n_{u_{a}}}^{2}=-150 \mathrm{dBW} / \mathrm{Hz}$, and $\sigma_{n_{u_{I D}}}^{2}=-110 \mathrm{dBW} / \mathrm{Hz}$. The backscattering efficiency coefficient is chosen as $\eta=0.78$ (corresponding to $1.1 \mathrm{~dB}$ loss [17]). The constants corresponding to the non-linear $\mathrm{EH}$ circuit are chosen as $\mathcal{E}^{\prime}=2.8 \mathrm{~mJ}, \alpha=1500$, and $\beta=0.0022$ [12], [13]. The harvested energy demand is assumed to be $\xi=-70 \mathrm{dBW}$, and the weighing factors are chosen as $\omega_{R}=1$ units/Gbit, $\omega_{E}=1$ units/nJ, and $\omega_{P}=1$ units/W. We use two test cases for the data 


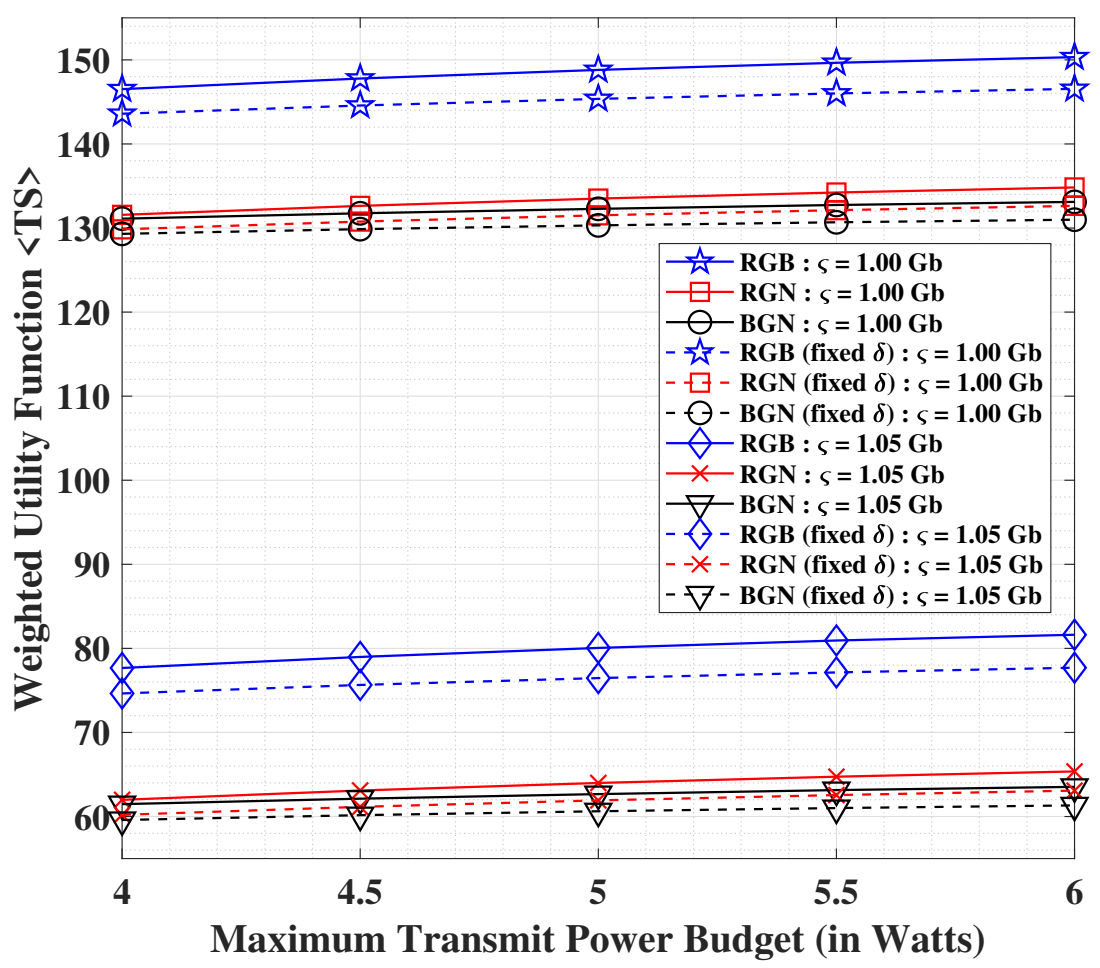

Fig. 3: Performance analysis of weighted utility function for TS scheme versus the maximum limitation on the total transmit power.

demands of $\varsigma=1.00$ Gbits and $\varsigma=1.05$ Gbits. As a benchmark scheme, we assume equal time distribution for both the hops in all the cases, i.e., fixed $\delta=0.5$. Furthermore, an average of 1000 random channel realizations is presented for each experiment.

In Fig. 2 and Fig. 3, we present the performance analyses of RGB, RGN and BGN in terms of the weighted utility function for the PS and TS schemes, respectively, versus the maximum limitation on the total transmit power. We observe that all the considered schemes can provide appreciable gains over their respective benchmark methods. The weighted utility function values for both PS and TS increases with growing overall maximum transmit power budget $\left(P^{\star}\right)$ of the system. On the other hand, the performance is adversely affected when the data demand is increased from $\varsigma=1.00$ Gbits to $\varsigma=1.05$ Gbits, which is due to the joint optimization in the weighted utility function. Particularly, this effect is due to the well-known rate-energy trade-off incurred because of the joint maximization of data and harvested energy along with the 
minimization of total transmit power. In the case of PS, we notice improvements of $10 \%$ and $12.5 \%$ for RGB over the RGN and BGN scenarios respectively for the data demand of $\varsigma=1.00$ Gbits, and improvements of $2 \%$ and $4 \%$ respectively for the data demand of $\varsigma=1.05$ Gbits, with an average decrement of $53 \%$ for a transition from former to the latter demand. Concerning the TS, we observe gains of $25 \%$ and $28 \%$ for RGB over the RGN and BGN scenarios respectively for the data demand of $\varsigma=1.00$ Gbits, and improvements of $11.5 \%$ and $12.5 \%$ respectively for the data demand of $\varsigma=1.05$ Gbits, with an average overall decrement of $80.5 \%$ for a transition from $\varsigma=1.00$ Gbits to $\varsigma=1.05$ Gbits. Clear benefits for RGB are observed over the RGN and BGN counterparts, with PS scheme outperforming the TS for all cases.

\section{CONCLUSIONS}

In this letter, we investigated a novel hybrid backscatter-and-relaying scheme to facilitate SWIPT in a $5 \mathrm{G}$ and beyond/6G Greencom environment, where the end-users are capable of decoding information and harvesting energy simultaneously, according to the PS and TS schemes while using the non-linear EH device for the latter. We formulated an optimization problem to jointly address the respective demands of data throughput, harvested energy and total transmit power, under some QoS constraints. We showed significant benefits of adopting RGB over the RGN and BGN counterpart systems with the help of numerical results, where the PS scheme was found to outperform the TS concerning the SWIPT at the end-user.

\section{REFERENCES}

[1] H. Viswanathan and P. E. Mogensen, "Communications in the 6G Era," IEEE Access, vol. 8, pp. 57063-57074, 2020.

[2] T. S. Rappaport and et al., "Overview of Millimeter Wave Communications for Fifth-Generation (5G) Wireless NetworksWith a Focus on Propagation Models,” IEEE Trans. Antennas Propag., vol. 65, no. 12, pp. 6213-6230, 2017.

[3] G. Yang, Q. Zhang, and Y.-C. Liang, "Cooperative ambient backscatter communications for green Internet-of-Things," IEEE Internet Things J., vol. 5, no. 2, pp. 1116-1130, 2018.

[4] J. N. Laneman, D. N. C. Tse, and G. W. Wornell, "Cooperative diversity in wireless networks: Efficient protocols and outage behavior," IEEE Trans. Inf. Theory, vol. 50, no. 12, pp. 3062-3080, December 2004.

[5] T. Cover and A. E. Gamal, "Capacity theorems for the relay channel," IEEE Trans. Inf. Theory, vol. 25, no. 5, pp. 572-584, September 1979.

[6] X. Zhou, R. Zhang, and C. K. Ho, "Wireless Information and Power Transfer: Architecture Design and Rate-Energy Tradeoff," IEEE Trans. Commun., vol. 61, no. 11, pp. 4754-4767, November 2013.

[7] B. Lyu, Z. Yang, H. Guo, F. Tian, and G. Gui, "Relay Cooperation Enhanced Backscatter Communication for Internetof-Things," IEEE Internet Things J., vol. 6, no. 2, pp. 2860-2871, 2019. 
[8] S. Gong and et al., "Backscatter Relay Communications Powered by Wireless Energy Beamforming," IEEE Trans. Commun., vol. 66, no. 7, pp. 3187-3200, 2018.

[9] S. Gong and et al., "Capitalizing Backscatter-Aided Hybrid Relay Communications with Wireless Energy Harvesting," IEEE Internet Things J., pp. 1-1, 2020.

[10] B. Lyu, D. T. Hoang, and Z. Yang, "Backscatter Then Forward: A Relaying Scheme for Batteryless IoT Networks," IEEE Wireless Commun. Lett., vol. 9, no. 4, pp. 562-566, 2020.

[11] N. V. Huynh and et al., “Ambient Backscatter Communications: A Contemporary Survey," IEEE Commun. Surveys Tuts., vol. 20, no. 4, pp. 2889-2922, 2018.

[12] J. Guo, H. Zhang, and X. Zhu, "Theoretical analysis of RF-DC conversion efficiency for class-F rectifiers," IEEE Trans. Microw. Theory Techn., vol. 62, no. 4, pp. 977-985, 2014.

[13] S. Gautam, E. Lagunas, S. Chatzinotas, and B. Ottersten, "Relay Selection and Resource Allocation for SWIPT in MultiUser OFDMA Systems,” IEEE Trans. Wireless Commun., vol. 18, no. 5, pp. 2493-2508, 2019.

[14] S. Boyd, S. P. Boyd, and L. Vandenberghe, Convex optimization, Cambridge university press, 2004.

[15] "MATLAB Optimization Toolbox (fmincon)," 9.7.0.1296695 (R2019b) Update 4, The MathWorks, Natick, MA, USA.

[16] P. Series, "Propagation data and prediction methods for the planning of short-range outdoor radiocommunication systems and radio local area networks in the frequency range $300 \mathrm{MHz}$ to $100 \mathrm{GHz}$," tech. rep., ITU, Tech. Rep. ITU-R, 2017.

[17] S. H. Kim and D. I. Kim, "Hybrid Backscatter Communication for Wireless-Powered Heterogeneous Networks," IEEE Trans. Wireless Commun., vol. 16, no. 10, pp. 6557-6570, 2017. 